\title{
Singing-Related Neural Activity Distinguishes Two Putative Pallidal Cell Types in the Songbird Basal Ganglia: Comparison to the Primate Internal and External Pallidal Segments
}

\author{
Jesse H. Goldberg, ${ }^{1}$ Avital Adler, ${ }^{2,3}$ Hagai Bergman, ${ }^{2,3}$ and Michale S. Fee ${ }^{1}$ \\ ${ }^{1}$ McGovern Institute for Brain Research, Department of Brain and Cognitive Sciences, Massachusetts Institute of Technology, Cambridge, Massachusetts \\ 02139, and ${ }^{2}$ Department of Medical Neurobiology (Physiology) and ${ }^{3}$ Interdisciplinary Center for Neural Computation, The Hebrew University-Hadassah \\ Medical School, Jerusalem 91120, Israel
}

The songbird area $\mathrm{X}$ is a basal ganglia homolog that contains two pallidal cell types-local neurons that project within the basal ganglia and output neurons that project to the thalamus. Based on these projections, it has been proposed that these classes are structurally homologous to the primate external (GPe) and internal (GPi) pallidal segments. To test the hypothesis that the two area X pallidal types are functionally homologous to GPe and GPi neurons, we recorded from neurons in area X of singing juvenile male zebra finches, and directly compared their firing patterns to neurons recorded in the primate pallidus. In area X, we found two cell classes that exhibited high firing $(\mathrm{HF})$ rates $(>60 \mathrm{~Hz})$ characteristic of pallidal neurons. HF-1 neurons, like most GPe neurons we examined, exhibited large firing rate modulations, including bursts and long pauses. In contrast, HF-2 neurons, like GPi neurons, discharged continuously without bursts or long pauses. To test whether HF-2 neurons were the output neurons that project to the thalamus, we next recorded directly from pallidal axon terminals in thalamic nucleus DLM, and found that all terminals exhibited singing-related firing patterns indistinguishable from HF-2 neurons. Our data show that singing-related neural activity distinguishes two putative pallidal cell types in area X: thalamusprojecting neurons that exhibit activity similar to the primate GPi, and non-thalamus-projecting neurons that exhibit activity similar to the primate GPe. These results suggest that song learning in birds and motor learning in mammals use conserved basal ganglia signaling strategies.

\section{Introduction}

The basal ganglia (BG) are an evolutionarily conserved set of brain nuclei widely implicated in motor control and learning (Smeets et al., 2000). The songbird area X, for example, is a specialized striatopallidal nucleus homologous to the mammalian basal ganglia (Fig. 1 A,B) (Farries and Perkel, 2002; Doupe et al., 2005). A recent study suggests that, like the mammalian BG, area X has two populations of pallidal neurons-(1) output neurons that send a long-range GABAergic projection to the thalamus and (2) local neurons that do not project to the thalamus but instead connect within the basal ganglia (Fig. 1B) (Farries et al., 2005). The projection patterns of these two populations are similar to neurons in the internal (GPi) and external (GPe) segments of the primate globus pallidus, consistent with

\footnotetext{
Received Jan. 11, 2010; revised March 11, 2010; accepted March 16, 2010.

Funding to M.S.F. was provided by the National Institutes of Health (Grant R01DC009183) and to J.H.G. by the Damon Runyon Research Foundation and Charles King Trust Postdoctoral Fellowships. Funding was provided to H.B. by an FP7 Select and Act grant and to A.A. by an Interdisciplinary Center for Neural Computation doctoral fellowship We thank Aaron Andalman, Dmitriy Aronov, and Michael Long for helpful comments.

Correspondence should be addressed to Michale S. Fee, McGovern Institute for Brain Research, Department of Brain and Cognitive Sciences, Massachusetts Institute of Technology, MIT 46-5133, 77 Massachusetts Avenue, Cambridge, MA 02139. E-mail: fee@mit.edu.

DOI:10.1523/JNEUROSCI.0168-10.2010

Copyright $\odot 2010$ the authors $\quad 0270-6474 / 10 / 307088-11 \$ 15.00 / 0$
}

mounting evidence that the division of the BG into direct and indirect pallidal pathways has existed for $>300$ million years, predating the division of birds, mammals, and reptiles into separate classes (Reiner, 2009).

It has yet to be determined whether the structural homology between the mammalian and non-mammalian BG extends to the functional signals that they generate during behavior. Most pallidal neurons in the primate exhibit a high baseline firing rate $(>60 \mathrm{~Hz})$ that distinguishes them from striatal cell types (DeLong, 1972). In addition, GPe and GPi neurons exhibit distinct activity in awake mammals-while most GPe neurons exhibit long pauses $(>300 \mathrm{~ms}$ ) and deep modulations in firing rate, most GPi neurons fire continuously, exhibit shallow modulations during movements, and do not generate long pauses (DeLong, 1971; Turner and Anderson, 1997; Elias et al., 2007).

Here we test the hypothesis that a similar distinction in pallidal firing patterns exists in the avian brain. We have recorded from two cell classes in area $\mathrm{X}$ that exhibited high firing $(\mathrm{HF})$ rates $(>60 \mathrm{~Hz})$ characteristic of pallidal neurons, and report that one group of HF neurons (HF-1) generated novel singing-related bursts and long pauses, while a second group (HF-2) fired continuously and never generated bursts or long pauses, similar to a cell type previously observed (Hessler and Doupe, 1999). We 
A

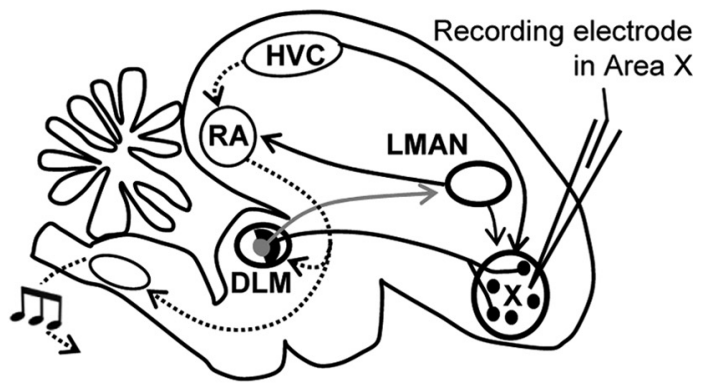

B

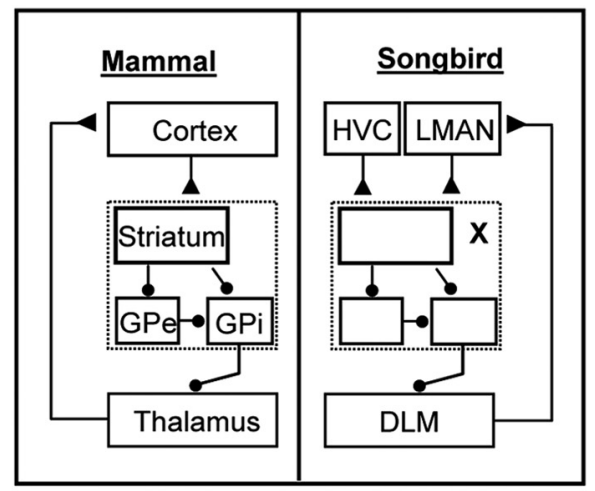

C

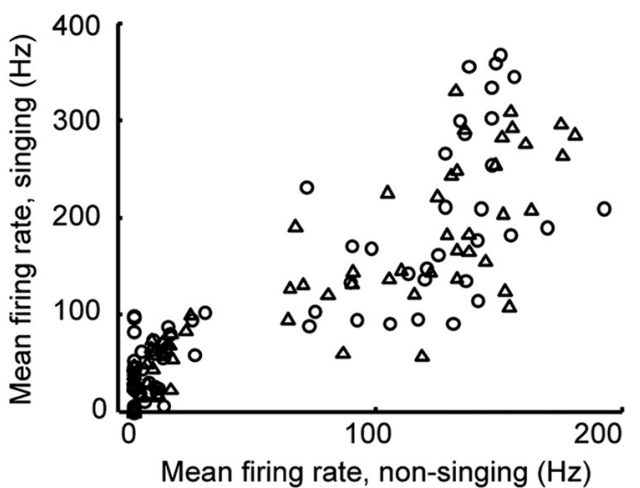

D

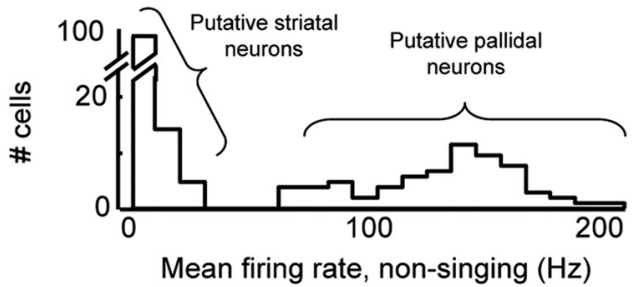

Figure 1. Nonsinging firing rate distinguishes high- and low-frequency cell classes in area $X$. $A$, Schematic of the avian song circuit showing the AFP (solid lines) and motor pathway (dotted lines). $\boldsymbol{B}$, The AFP is homologous to a mammalian BG-thalamocortical loop. Like the mammalian $B G$, area $X$ contains striatal neurons and two pallidal cell classes: one class that projects within the basal ganglia — possibly homologous to the primate GPe — and a second class that projects to the thalamus - homologous to the primate GPi. The AFP appears to lack a homolog of the STN, which is interposed between cortex, striatum, and pallidus in the mammalian BG (omitted in this figure). $C$, Mean rate during singing is plotted against the mean rate during nonsinging epochs for each unit recorded in area $X$. Triangles and circles represent data from subsong and plastic song birds, respectively. D, Histogram of nonsinging firing rates for all units recorded in area $X$ reveals a distinct population of high-firing-rate neurons.

developed a quantitative analysis of firing rate modulations that cleanly separated these two classes of neurons.

To determine whether one cell class constituted the neurons that project to the thalamus, we next recorded directly from the pallidal axon terminals of area $\mathrm{X}$ outputs in the thalamic nucleus DLM (Person and Perkel, 2007), and found that all terminals exhibited singing-related firing patterns quantitatively indistinguishable from HF-2 neurons.

Qualitatively, the firing pattern of HF-1 neurons, including long pauses and deep modulations in firing rate, was reminiscent of published reports of GPe neuronal activity, while the activity of HF-2 neurons and pallidal terminals resembled that of GPi neurons (DeLong, 1971). To make a quantitative comparison, we next analyzed 129 neurons recorded in the primate pallidus and found that the analysis that separated HF-1 from HF-2 neurons also distinguished most GPe neurons from GPi neurons. These data suggest that the distinct signals generated by two pallidal pathways are evolutionarily conserved aspects of the BG circuit, and that motor learning in birds and mammals uses common, and ancient, signaling strategies.

\section{Materials and Methods}

Finches. Subjects were 21 juvenile male zebra finches, $36-70 \mathrm{~d}$ post-hatch (dph). Birds were obtained from the Massachusetts Institute of Technology (MIT) zebra finch breeding facility (Cambridge, MA). The care and experimental manipulation of the animals were performed in accordance with guidelines of the National Institutes of Health and were reviewed and approved by the MIT Committee on Animal Care.

Songbird data acquisition. Neural recordings were performed using a motorized microdrive described previously (Fee and Leonardo, 2001). Cells in DLM and area X were isolated by searching for spontaneous or antidromically evoked spiking activity; units had signal-to-noise ratios (peak spike amplitude compared to SD of noise) greater than 15:1. Antidromic identification of LMAN-projecting DLM neurons was performed with a bipolar stimulating electrode implanted in LMAN using techniques described previously (Hahnloser et al., 2002). Data were acquired and analyzed using custom Matlab software (A. Andalman, D. Aronov, and J.H.G.). Small electrolytic lesions (20 $\mu \mathrm{A}$ for $15 \mathrm{~s}$ ) were made through the electrodes at the conclusion of experiments for histological verification of electrode position. To visualize pallidal terminals in DLM (as in Fig. 3B), Alexa 555-dextran (molecular weight, 10,000; Molecular Probes) was injected into area X, for anterograde labeling of calyceal terminals, and Alexa 647-dextran into LMAN for retrograde labeling of DLM neurons. Brain slices $(100 \mu \mathrm{m})$ were imaged with a Nikon PCM2000 confocal microscope, and ImageJ was used to merge serial sections.

Data analysis. Spikes were sorted offline using custom Matlab software. Spike widths were computed as the full width at half maximum of the negative-going deflection of the average of 50 spike waveforms (supplemental Fig. 1, available at www.jneurosci.org as supplemental material). We represented neural activities as instantaneous firing rates (IFRs), $R(t)$, defined at each time point as the inverse of the enclosed interspike interval as follows (Eq. 1):

$$
R(t)=\frac{1}{t_{i+1}-t_{i}}, \quad \text { for } t_{i}<t \leq t_{i+1}
$$

where $t_{i}$ is the time of the $i$ th spike. To compute the power spectra of the IFRs, IFR signals were mean-subtracted and multiplied by a Hanning window before calculating the fast Fourier transform.

To analyze the modulations in firing rate, in particular to distinguish HF-1 neurons from HF-2 neurons, we smoothed the IFR signals with a finite impulse response (FIR) equiripple filter with a passband below 25 $\mathrm{Hz}$ and stopband greater than $75 \mathrm{~Hz}$, with $80 \mathrm{~dB}$ attenuation in the stopband. To remove the DC offset (i.e., the high average firing rate), IFRs were mean-subtracted using an infinite impulse response (IIR) $1 \mathrm{~Hz}$ high-pass filter generated with coefficients $\boldsymbol{b}=\left[\begin{array}{ll}1-1 & -1\end{array}\right]$ and $\boldsymbol{a}=[1$ -0.9988] (supplemental Fig. $2 A$, available at www.jneurosci.org as supplemental material). Bursts were defined as peaks in the smoothed IFR signal that exceeded 250 spikes per second.

For the primate GP neurons, which exhibited slower modulations, IFRs were smoothed with a FIR equiripple filter with a passband below 2 
$\mathrm{Hz}$ and a stopband greater than $4 \mathrm{~Hz}$, with $80 \mathrm{~dB}$ attenuation in the stopband. IFRs were mean-subtracted using an IIR $0.1 \mathrm{~Hz}$ high-pass filter generated with coefficients $\boldsymbol{b}=[1-1]$ and $\boldsymbol{a}=[1-0.9995]$ (supplemental Fig. $2 B$, available at www.jneurosci.org as supplemental material). Bursts were defined as peaks in the smoothed IFR that exceeded 50 spikes per second. Burst thresholds were defined to optimally separate distinct classes of neurons, and in both songbirds and primates could be varied $\pm 20 \%$ without significant changes to the results.

To determine the time at which terminal and HF-2 activity increased before song onsets, we averaged the instantaneous firing rates during the $3 \mathrm{~s}$ that preceded song onsets, and used the first $500 \mathrm{~ms}$ of this epoch as baseline. The averaged trace was smoothed with a $25 \mathrm{~ms}$ Gaussian filter. The onset of the presinging increase in activity was estimated as the point at which the smoothed trace exceeded 2 SDs from baseline. The time for pallidal activity to return to baseline was determined as above, using the 3 s epoch following song offsets.

Interbout epochs were defined as silent periods separated from singing by $<5 \mathrm{~s}$. Note that the interbout epoch includes both the seconds immediately preceding bout onset, and following bout offset. Although bursting in the seconds following bout offset appeared to be a prominent feature of HF-1 neurons, there were in fact no statistical differences between burst rate or burst characteristics between the immediate postbout period ( $<2 \mathrm{~s}$ following bout offset) and all interbout epochs combined. Nonsinging firing rates were calculated from silent periods separated from singing by $>10 \mathrm{~s}$.

Analysis of correlations of neural activity to song temporal structure. For plastic song birds, syllables were identified both manually and with an automated clustering algorithm (A. Andalman). The songs of the plastic song birds in this study were not yet organized into motifs-thus we analyzed correlations on a syllable-by-syllable basis. We included in this analysis only those birds with song repertoires that included distinct, identifiable syllables ( 67 syllables for HF-2 neurons, 82 syllables for terminals; 45 syllables for HF-1 neurons), and only analyzed cells that were recorded for $>30$ syllable renditions (mean \pm SD number syllables per neuron $=195 \pm 207$, range $=31-1337$ ). Neural activity from $60 \mathrm{~ms}$ before syllable onsets to $60 \mathrm{~ms}$ following the median syllable duration was linearly time warped, using as a reference the syllable's median duration (supplemental Fig. 6, available at www.jneurosci.org as supplemental material), as previously described (Olveczky et al., 2005; Kao et al., 2008). Syllables of subsong birds ( $<45 \mathrm{dph}$ ) are highly unstructured, and neural correlations to their acoustic or temporal structure are outside the scope of this study.

To determine the significance of firing rate peaks and minima within syllables, a rate histogram ( $10 \mathrm{~ms}$ bin size) was generated for the syllablealigned spike trains. Surrogate histograms were also calculated for all spike trains after a random time shift, as described previously (Olveczky et al., 2005). The shift was circular, such that spikes wrapped around to the beginning of the syllable; time shifts were chosen randomly from a uniform distribution with the width of the syllable plus $120 \mathrm{~ms}(60 \mathrm{~ms}$ before and after syllable). Peaks and minima in firing rate were considered significant when they crossed the 1st (for minima) and 99th (for peaks) percentile of the compiled minima and maxima of the surrogate histograms. Modulation depths were computed for each syllable, and for each neuron, and were defined as the difference between the peak and the minimum of the rate histogram ( $10 \mathrm{~ms}$ bin size as above), divided by the mean rate during that syllable (Fee et al., 1997).

To quantify trial-to-trial differences in neural activity on short timescales, we computed the average correlation coefficient (CC) between the instantaneous firing rate for all pairs of trials, as follows (Eqs. 2, 3):

$$
\begin{aligned}
\mathrm{CC} & =\frac{1}{N_{\text {pairs }}} \sum_{j>i}^{N_{\text {trials }}} \mathrm{CC}_{i j} \\
\mathrm{CC}_{i j} & =\frac{\left\langle\hat{r}_{i}(t) \hat{r}_{j}(t)\right\rangle_{t}}{\sqrt{\left\langle\hat{r}_{i}(t)^{2}\right\rangle_{t}\left\langle\hat{r}_{j}(t)^{2}\right\rangle_{t}}},
\end{aligned}
$$

where $\hat{r}(t)$ was computed by smoothing spike trains with a Gaussian kernel of 10 ms SD (Olveczky et al., 2005; Kao et al., 2008). To determine the statistical significance of the correlation, we also compared the correlation distributions to those calculated after random time shifts were added to the spike trains, as described above (Olveczky et al., 2005). In all neurons, independent of cell class, the correlation distributions were significantly different from those of the randomly shuffled spike trains $(p<0.01$, Kolmogorov-Smirnov test). Finally, we also calculated CCs on non-time-warped syllables, resulting in, on average, a 0.005 decrease in CC value (supplemental Fig. 6, available at www.jneurosci.org as supplemental material).

For the analyses examining trial-to-trial variability in pause or burst times in HF-1 neurons, pauses were defined as intervals greater than the 99th percentile interspike interval (ISI), and bursts were defined as described above. For each syllable that contained at least one pause (or burst), we generated a vector of zeros and ones, where time within each pause (or burst) equaled 1 . This vector was then de-meaned and used as the $\hat{r}(t)$ in Equations 2 and 3 to generate a distribution of correlation coefficients. To determine the significance of the resulting distribution, we performed a Kolmogorov-Smirnov test on a surrogate distribution of CCs generated from randomly shuffled pause (or burst) times. For this analysis, pauses and bursts were analyzed separately.

Analysis of simultaneously recorded pairs of terminals and HF-2 neurons. Each microdrive was equipped with three electrodes, spaced $\sim 300 \mu \mathrm{m}$ apart, enabling on rare occasions the simultaneous recording of pairs. Two pairs of HF-2 and two pairs of terminals were recorded. Each unit within each pair had $>3500$ spikes under each condition. Raw crosscorrelograms for singing and nonsinging periods were constructed for each pair as rate histograms of spike times of neuron 2 aligned to spike times of neuron 1 . To determine the significance of peaks and minima in the cross-correlograms, 1000 surrogate cross-correlograms of independent spike trains were generated for each pair, under each condition, by randomly phase shifting one of the spike trains, preserving its overall spike statistics, as described above. Peaks (or minima) in the true crosscorrelograms were considered significant if they crossed the 1st (for minima) or 99th (for peaks) percentile of the compiled minima and maxima of the surrogate histograms.

Primate data acquisition. All experimental protocols were conducted in accordance with the National Institutes of Health Guide for the Care and Use of Laboratory Animals and the Hebrew University guidelines for the use and care of laboratory animals in research. The experimental protocols were approved and supervised by the Institutional Animal Care and Use Committee of the Hebrew University and Hadassah Medical Center. The Hebrew University is accredited by the Association for Assessment and Accreditation of Laboratory Animal Care International. Methods were given in detail in previous manuscripts (Joshua et al., 2009). A brief summary of these methods is given here.

One monkey (L; Macaque fascicularis, female $4 \mathrm{~kg}$ ) was used in this study. During recording sessions, monkey L was engaged in a probabilistic delay classical-conditioning task. The monkey's head was immobilized and eight glass-coated tungsten microelectrodes were advanced separately (EPS, Alpha-Omega Engineering) in a coronal plane, $50^{\circ}$ from the axial plane into the GPe or the GPi. Two experimenters controlled the position of the eight electrodes and the real-time spike sorting (AlphaMap, ASD, Alpha-Omega Engineering). Recorded spike trains were subjected to an offline quality analysis, which included tests for discharge rate stability, refractory period, spike waveform isolation, and recording time. GPe neurons were identified according to their stereotaxic coordinates (based on MRI and primate atlas data) and their physiological identification. The physiological parameters included the characteristic symmetric, narrow, and high-amplitude spike waveform; the typical firing rate and pattern characterized by high-frequency discharge rates ( $>20 \mathrm{~Hz}$ ) interrupted by long intervals of total silence (DeLong, 1971); and the preceding neuronal activity of the striatum obtained in the same electrode trajectory to the GPe. GPe low-frequency discharge bursting neurons, which may represent a different population of GPe neurons, were excluded from the current data base. The identification criteria for GPi neurons included, apart from stereotaxic coordinates (based on MRI and primate atlas data), the depth of the electrode; the physiological identification of border cells between the GPe and the GPi; and the real time assessment of the firing pattern of the cell. Pallidal recordings were 
A
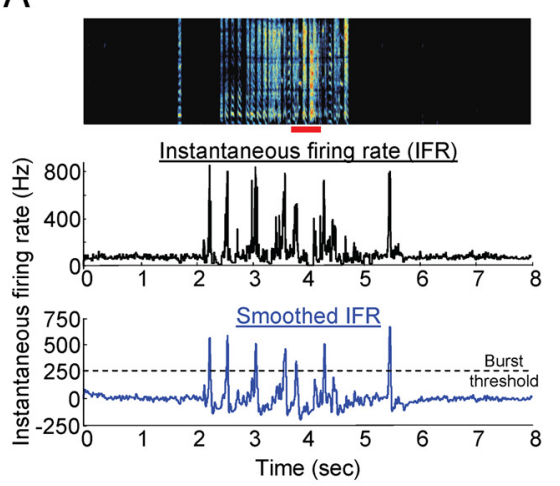

$E$

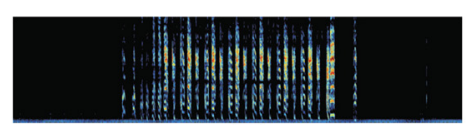

Instantaneous firing rate (IFR)



I

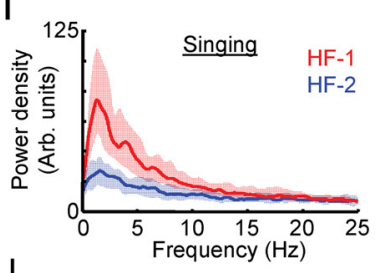

$J$

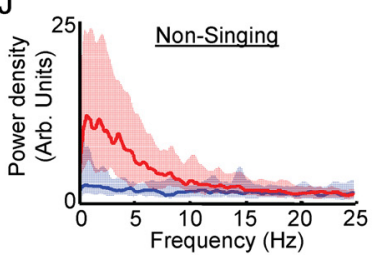

$\mathrm{K}$
B

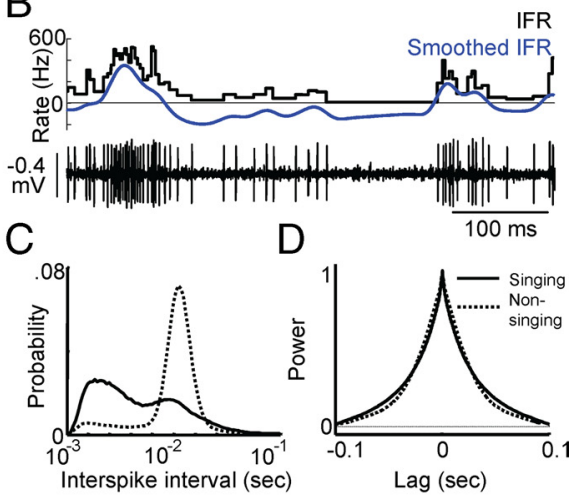

$\mathrm{F}$

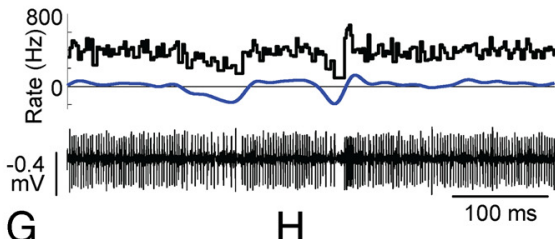

$\mathrm{G}$
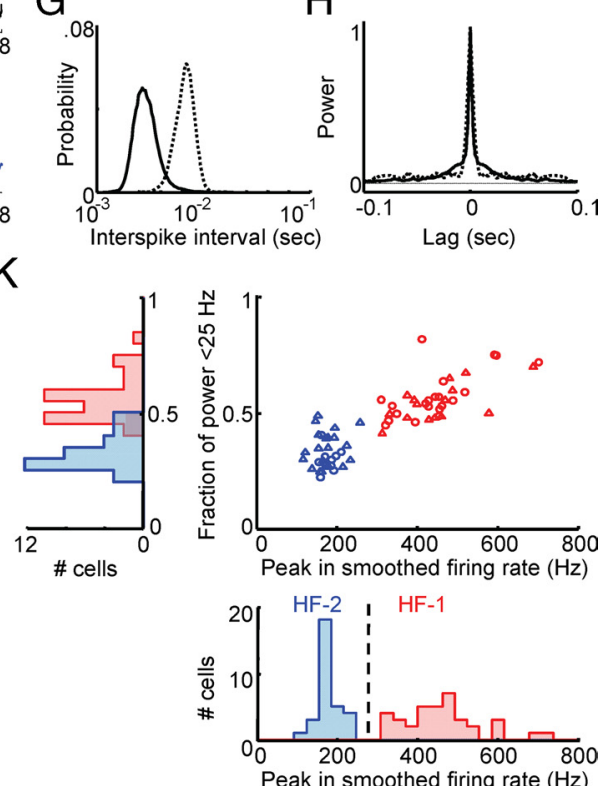

Figure 2. Singing-related neural activity distinguishes two classes of HF neurons in area X. A-D, Example of an HF-1 neuron. $A$, Representative spectrogram plotted above the IFR computed from the spikes of a single neuron (top), and the same IFR trace smoothed by low-pass filtering below $25 \mathrm{~Hz}$. The high mean firing rate was removed (mean-subtracted) using a high-pass filter above $1 \mathrm{~Hz}$ (see Materials and Methods). Bursts were detected as peaks in the mean-subtracted, smoothed IFR that exceeded 250 spikes per second (dotted line). $\boldsymbol{B}$, Expanded view of the IFR (black) and the smoothed IFR (blue), and the raw voltage data from the period indicated by the red bar beneath the spectrogram in $A$. $C, D$, The log of the ISI distributions $(\boldsymbol{C})$ and the lag-autocorrelation function of the IFR ( $\boldsymbol{D}$ ) are plotted for data from singing and nonsinging periods (solid and dotted lines, respectively) for the neuron shown in $\boldsymbol{A}$. $\boldsymbol{E}-\boldsymbol{H}$, Data are plotted exactly as in $\boldsymbol{A}-\boldsymbol{D}$, for a neuron classified as HF-2.I,, , The power spectra of the IFRs (median over all cells, shaded areas indicate 25 th to 75 th percentile) for $37 \mathrm{HF}-1$ neurons (red) and $33 \mathrm{HF}-2$ neurons (blue), for data from singing periods $(\boldsymbol{I})$ and from nonsinging periods $(\boldsymbol{J})$. Note that $\mathrm{HF}-1$ neurons have more power at low frequencies $(<25 \mathrm{~Hz}$ ). $\boldsymbol{K}$, For each HF-1 neuron (red) and HF-2 neuron (blue) recorded in area X, the percentage of the IFR power spectrum at low frequencies is plotted against the peak (99th percentile) of the mean-subtracted, smoothed IFR. Triangles and circles are data from subsong and plastic song birds, respectively. At bottom, the histogram of peak values of the mean-subtracted, smoothed IFR distinguishes two classes of HF neurons. Neurons with peaks greater than 250 spikes per second were defined as HF-1 neurons. At left, the histogram of percentage of IFR power spectrum at frequencies less than $25 \mathrm{~Hz}$.

not restricted to specific areas within the GPe and GPi, but spanned the entire nuclei.

\section{Results}

For area X recordings, we recorded 185 neurons from 10 juvenile birds, aged 40-70 dph. In awake, nonsinging birds, we found that

the average firing rate of area $\mathrm{X}$ neurons fell into two main classes: high-frequency (HF) neurons with average nonsinging firing rates greater than $60 \mathrm{~Hz}(127 \pm 30$ $\mathrm{Hz}, n=70$, all error bars indicate SD), and low-frequency neurons with nonsinging rates less than $30 \mathrm{~Hz}(4.9 \pm 6.3 \mathrm{~Hz}, n=$ 115) (Fig. $1 C, D$ ). We hypothesize that the high and low firing cell classes represent the pallidal and striatal neuronal cell classes, respectively, that are known to reside in area X (Farries and Perkel, 2002). This hypothesis is based on previous studies of area $\mathrm{X}$ neurons in brain slice, which revealed high spontaneous firing rates in pallidal neurons and low rates in striatal neurons (Farries and Perkel, 2002), and in vivo, which found that HF neurons in area X project to DLM (Leblois et al., 2009), as well as on numerous reports in mammals demonstrating high pallidal and low striatal firing rates in awake animals (DeLong, 1972; Johnstone and Rolls, 1990; Bar-Gad et al., 2003). In the present study, we focus exclusively on the HF neurons. The putative striatal neurons were the subject of a different study (Goldberg and Fee, 2010).

\section{Singing-related neural activity}

distinguishes two classes of HF neurons We observed two different types of singing-related firing patterns among HF neurons. Some HF neurons exhibited prominent bursts and long pauses (Fig. $2 A, B)$, while others discharged continuously (Fig. 2E,F). These differences in spiking behavior were readily apparent in the IFR (see Materials and Methods), where bursting and pausing were associated with large modulations in firing rate (Fig. 2A,B). We found, by quantifying the modulations of the IFR (Fig. $2 A-J$ ), that HF neurons in area $X$ could be separated into two distinct cell classes: those that generated large peaks in a smoothed version of the IFR (defined as HF-1), and those that did not (defined as HF-2) (Fig. $2 \mathrm{~K}$ ) (see Materials and Methods).

Note that the large firing rate fluctuations of HF-1 neurons occurred preferentially at low modulation frequencies $(<25$ $\mathrm{Hz}$ ), such that the power spectrum of the IFRs of HF-1 neurons showed higher power at low frequencies than did HF-2 neurons (Fig. $2 I$, J, see Fig. $4 L$ ). In contrast, HF-2 neurons showed more fluctuations in firing rate at modulation frequencies above $35 \mathrm{~Hz}$ (see Fig. 4L). Although the total fraction of power below $25 \mathrm{~Hz}$ largely separated HF-1 and HF-2 neurons (Fig. $2 \mathrm{~K}$ ), the best separation of HF neurons into two distinct clusters was obtained by examining the smoothed IFR traces (see Materials and Methods). It can be seen that, in the smoothed IFR, HF-1 neurons exhibited bursts with 
Table 1. Singing and nonsinging spiking statistics for HF neurons in area $X$ and pallidal terminals recorded in DLM

\begin{tabular}{|c|c|c|c|c|c|c|c|c|c|c|}
\hline & $\begin{array}{l}\text { Rate, } \\
\text { nonsinging } \\
(\mathrm{Hz})\end{array}$ & $\begin{array}{l}\text { Rate, } \\
\text { singing } \\
(\mathrm{Hz})\end{array}$ & $\begin{array}{l}\text { C.V., } \\
\text { nonsinging }\end{array}$ & $\begin{array}{l}\text { C.V., } \\
\text { singing }\end{array}$ & $\begin{array}{l}\text { 99th } \\
\text { percentile ISI, } \\
\text { singing (ms) }\end{array}$ & $\begin{array}{l}\text { 99th } \\
\text { percentile ISI, } \\
\text { nonsinging }\end{array}$ & $\begin{array}{l}\text { IFR autocorrelation } \\
\text { half-width (ms), } \\
\text { singing }\end{array}$ & $\begin{array}{l}\text { IFR autocorrelation } \\
\text { half-width (ms), } \\
\text { nonsinging }\end{array}$ & $\begin{array}{l}\% \text { of IFR } \\
\text { spectrum in } \\
\text { the } 1-25 \mathrm{~Hz} \\
\text { band,singing }\end{array}$ & $\begin{array}{l}\% \text { of IFR spectrum } \\
\text { in the } 1-25 \mathrm{~Hz} \\
\text { band, nonsinging }\end{array}$ \\
\hline HF-1, subsong $(n=16)$ & $120.0 \pm 30.1$ & $135.8 \pm 34.1^{*}$ & $0.39 \pm 0.10$ & $0.86 \pm 0.25^{*}$ & $37.5 \pm 24.7^{*}$ & $19.7 \pm 7.9$ & $14.1 \pm 7.6$ & $20.1 \pm 14.0^{*}$ & $8.8 \pm 1.4$ & $9.1 \pm 2.1$ \\
\hline HF-1, plastic $(n=21)$ & $121.7 \pm 29.8$ & $142.6 \pm 44.4^{*}$ & $0.48 \pm 0.36$ & $0.95 \pm 0.36^{*}$ & $41.1 \pm 26.5^{*}$ & $22.9 \pm 15.5$ & $16.8 \pm 9.8$ & $25.6 \pm 14.7^{*}$ & $9.1 \pm 1.7$ & $9.8 \pm 1.9$ \\
\hline HF-2, subsong $(n=22)$ & $131.4 \pm 34.1$ & $232.5 \pm 60.8^{*}$ & $0.32 \pm 0.12$ & $0.49 \pm 0.07^{*}$ & $12.6 \pm 5.4$ & $19.0 \pm 15.8^{*}$ & $5.4 \pm 1.7$ & $7.1 \pm 2.4^{*}$ & $5.8 \pm 1.1$ & $7.0 \pm 1.9$ \\
\hline HF-2, plastic $(n=11)$ & $136.0 \pm 23.2$ & $308.6 \pm 46.0^{*}$ & $0.27 \pm 0.05$ & $0.42 \pm 0.06^{*}$ & $9.3 \pm 2.1$ & $13.2 \pm 4.6^{*}$ & $4.5 \pm 1.4$ & $7.1 \pm 2.4^{*}$ & $5.3 \pm 0.7$ & $6.5 \pm 1.6$ \\
\hline Terminals, subsong $(n=14)$ & $149.5 \pm 36.0$ & $296.0 \pm 54.0^{*}$ & $0.31 \pm 0.08$ & $0.47 \pm 0.09^{*}$ & $10.1 \pm 3.9$ & $13.0 \pm 3.1^{*}$ & $5.1 \pm 1.8$ & $7.6 \pm 2.1^{*}$ & $5.5 \pm 0.9$ & $6.7 \pm 1.2$ \\
\hline Terminals, plastic $(n=23)$ & $137.6 \pm 22.4$ & $286.2 \pm 59.2^{*}$ & $0.31 \pm 0.08$ & $0.47 \pm 0.11^{*}$ & $10.2 \pm 3.7$ & $14.3 \pm 2.6^{*}$ & $5.1 \pm 1.3$ & $6.9 \pm 1.2^{*}$ & $5.8 \pm 0.9$ & $6.7 \pm 0.9$ \\
\hline Interclass comparisons & n.s. & $\begin{array}{c}\mathrm{HF}-1<\mathrm{HF}-2, \\
\text { terminals }\end{array}$ & $\begin{array}{c}H F-1>H F-2, \\
\text { terminals }\end{array}$ & $\begin{array}{c}\mathrm{HF}-1>\mathrm{HF}-2 \text {, } \\
\text { terminals }\end{array}$ & $\begin{array}{c}\mathrm{HF}-1>\mathrm{HF}-2, \\
\text { terminals }\end{array}$ & $\begin{array}{l}\mathrm{HF}-1> \\
\quad \text { terminals }\end{array}$ & $\begin{array}{c}\mathrm{HF}-1>\mathrm{HF}-2, \\
\text { terminals }\end{array}$ & $\begin{array}{c}H F-1>H F-2, \\
\text { terminals }\end{array}$ & $\begin{array}{c}\mathrm{HF}-1>\mathrm{HF}-2, \\
\text { terminals }\end{array}$ & $\begin{array}{c}\mathrm{HF}-1>\mathrm{HF}-2, \\
\text { terminals }\end{array}$ \\
\hline
\end{tabular}

Asterisks indicate significant within-class differences of singing versus nonsinging data ( $p<0.01$, paired $t$ test). Interclass comparisons indicate significant differences between cell classes ( $p<0.01$, unpaired $t$ test). Data from subsong and plastic song birds were combined for comparisons across neuronal classes.

peak firing rates between 300 and 700 spikes per second, whereas HF-2 neurons exhibited peak firing rates between 100 and 250 spikes per second (Fig. $2 \mathrm{~K}$ ). Thus, peak firing rate in the smoothed IFR served as the metric by which HF-1 and HF-2 neurons were classified. We first discuss the firing patterns of the HF-1 cell class.

\section{HF-1 neurons exhibited singing-related high-frequency bursts and long pauses}

High-frequency bursts, defined as events where the band-passed IFR exceeded $250 \mathrm{~Hz}$ (see Materials and Methods), were a prominent feature in the firing pattern of HF-1 neurons (Fig. 2A,B). Bursts in HF-1 neurons occurred frequently during singing $\left(1.16 \pm 0.85 \mathrm{~s}^{-1}\right)$ and in the brief silent periods in between singing bouts $\left(0.58 \pm 0.31 \mathrm{~s}^{-1}\right.$, interbout epochs, see Materials and Methods), but much less frequently when the bird was not singing (nonsinging burst incidence: $0.24 \pm 0.24 \mathrm{~s}^{-1}$ ).

Bursting was associated with a prominent shoulder, and in some cases a second peak, in the short interval side of the ISI distributions of HF-1 neurons (Fig. 2C; supplemental Fig. 3B, available at www.jneurosci.org as supplemental material). In addition, because bursts consisted of multiple intervals generated in quick succession, they were associated with broad peaks in the instantaneous firing rate (Fig. $2 \mathrm{~A}$; supplemental Fig. $3 \mathrm{~A}$, available at www.jneurosci.org as supplemental material). Accordingly, the autocorrelation of the IFR exhibited a broad peak (full width at half maximum $=15.7 \pm 8.7 \mathrm{~ms}$ ) that was significantly wider than the average duration of a single interspike interval $(8.0 \pm 2.8$ ms, $p<0.0001$, paired $t$ test, $n=37$ neurons) (Fig. $2 D$ ).

Despite the incidence of high-frequency bursts during singing, this was not associated with a significant increase in the mean firing rate of HF- 1 neurons $(121 \pm 29 \mathrm{~Hz}$ nonsinging vs $135 \pm 42$ $\mathrm{Hz}$ singing, $p>0.09$ ) (supplemental Fig. $3 D$, available at www. jneurosci.org as supplemental material; Table 1). Between bursts, HF-1 neurons frequently exhibited long pauses in firing. As has been noted in some mammalian pallidal neurons, pauses were not discrete events, but represented the long intervals along the distribution of interspike intervals (ISIs) (Fig. 2C) (Elias et al., 2007). Thus, we defined pauses as intervals longer than the 99th percentile ISI during singing and found that they have an average duration of $53.1 \pm 35.0 \mathrm{~ms}$. In summary, HF-1 neurons did not fire continuously during singing - discharge was regularly interrupted by bursts and long pauses. Finally, the firing statistics of HF-1 neurons in plastic song and subsong birds were identical by all of these measures (Table 1).

\section{HF-2 neurons did not generate bursts or long pauses}

In contrast to HF-1 neurons, HF-2 neurons did not exhibit large peaks in the band-passed IFR (burst rate $=0.01 \pm 0.03 \mathrm{~s}^{-1}$ ) (Fig.
$2 E, F)$. Several features of the firing patterns of HF-2 neurons further distinguished them from HF-1 neurons. First, all HF-2 neurons significantly increased their firing rates during singing $(133 \pm 31 \mathrm{~Hz}$ nonsinging vs $258 \pm 66 \mathrm{~Hz}$ singing, $p<0.0001$, paired $t$ test $n=33$; 10 birds) (Fig. $2 E-G$; supplemental Fig. $3 H-K$, available at www.jneurosci.org as supplemental material). The firing rate increased in the hundreds of milliseconds before singing $(0.45 \pm 0.52 \mathrm{~s})$, and slowly returned to baseline following singing $(1.40 \pm 0.57 \mathrm{~s}$, see Materials and Methods). Second, during singing, HF-2 neurons exhibited substantially less variability in interspike interval duration than HF-1 neurons [HF-1: coefficient of variation (C.V.) $=0.90 \pm 0.32$; HF-2: C.V. $=0.48 \pm .09$, $p<0.001$ ] (supplemental Fig. 3, available at www.jneurosci.org as supplemental material; Table 1). Third, firing rate fluctuations in HF-2 neurons were extremely rapid, such that a given interspike interval was weakly correlated with its neighboring intervals, resulting in flat spike train autocorrelations (supplemental Fig. 4, available at www.jneurosci.org as supplemental material) and a sharp peak in the IFR autocorrelation. In fact, peaks in the IFR autocorrelation were significantly narrower in HF-2 neurons than HF-1 neurons during singing (full width at half maximum: $5.1 \pm 1.6 \mathrm{~ms}, p<0.001 t$ test vs HF-1) (Fig. $2 H$; supplemental Fig. $3 B$, available at www.jneurosci.org as supplemental material; Table 1), and in the range of the average duration of a single interspike interval $(4.2 \pm 1.4 \mathrm{~ms})$.

Finally, HF-2 neurons did not exhibit long pauses in firing. During singing, even intervals as short as $20 \mathrm{~ms}$ occurred rarely $\left(0.59 \pm 0.91 \mathrm{~s}^{-1}\right)$. In contrast, such intervals occurred 10 times as often in HF-1 neurons $\left(6.0 \pm 4.2 \mathrm{~s}^{-1}, p<0.001\right.$, unpaired $t$ test). In addition, singing-related pauses in HF-2 neurons (defined as intervals greater than the 99th percentile ISI) were significantly shorter than in HF-1 neurons (HF-2: $16.1 \pm 7.0 \mathrm{~ms}$; HF-1: $53.1 \pm$ $35.0 \mathrm{~ms} ; p<0.001$, unpaired $t$ test) (supplemental Fig. $3 B$, available at www.jneurosci.org as supplemental material; Table 1). Notably, the discharge of HF-2 neurons-continuous spiking with only brief fluctuations in rate, and without the generation of bursts or long pauses-is remarkably similar to the firing patterns reported in the mammalian GPi (DeLong, 1971). This led us to hypothesize that the HF-2 neurons constituted the area $\mathrm{X}$ output neurons that project to the thalamic nucleus DLM.

\section{Singing-related activity of pallidal axon terminals recorded in DLM was indistinguishable from HF-2 neurons}

To directly test this hypothesis, we implanted electrodes into DLM, where the large axon terminals of area $\mathrm{X}$ pallidal projection neurons can be recorded extracellularly (Fig. 3A,B) (Person and Perkel, 2007; Kojima and Doupe, 2009; Leblois et al., 2009). We recorded 66 units in DLM of 11 birds (10 juveniles, aged 36-70 

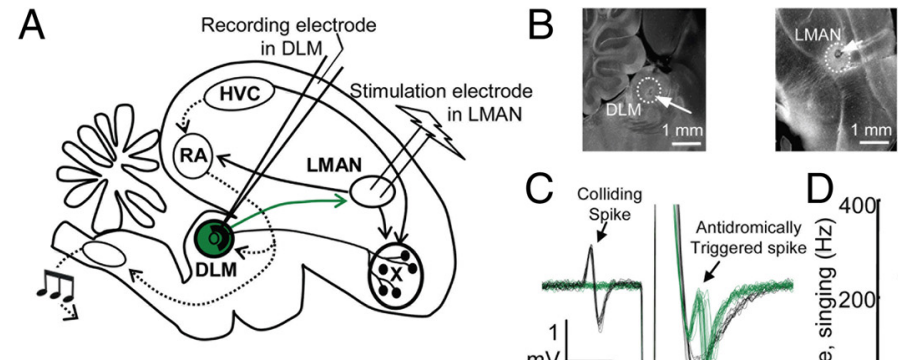

C Colliding
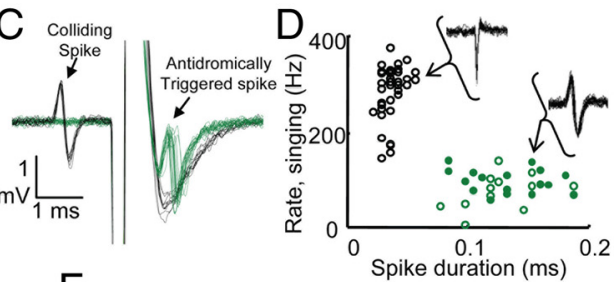

E

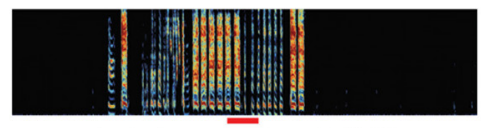

$\underline{\text { Instantaneous firing rate (IFR) }}$

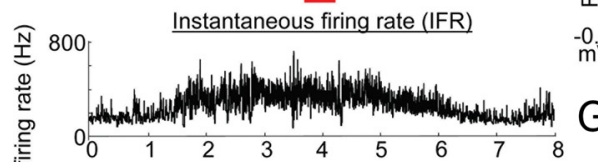

F
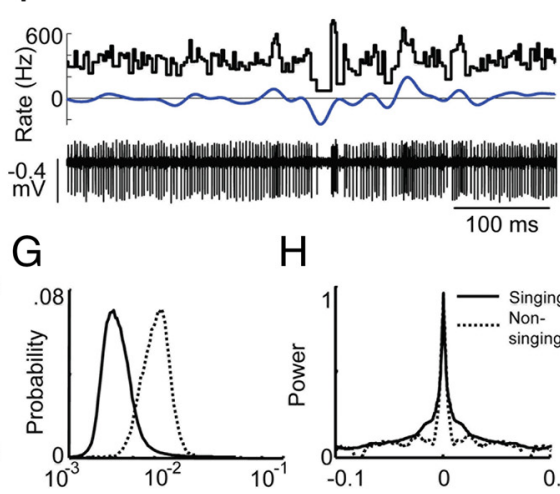

$\mathrm{H}$

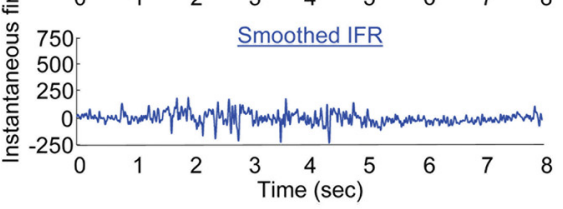

Interspike interval (s)

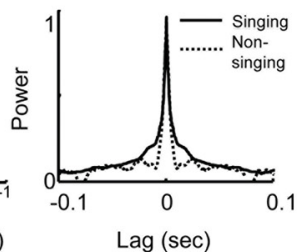

K
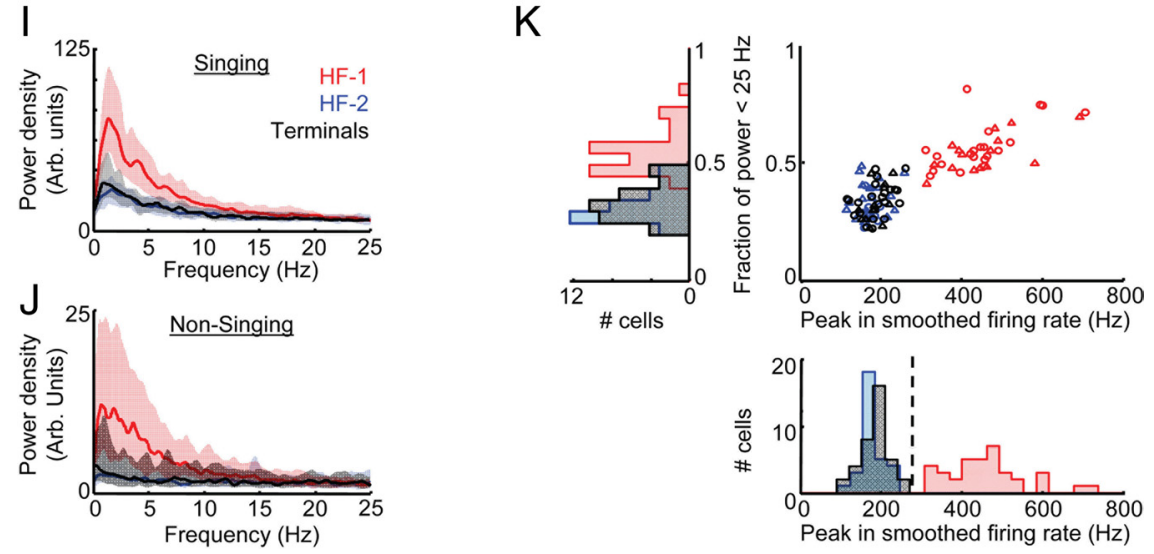

Figure 3. Pallidal axon terminals recorded in DLM exhibit singing-related firing patterns indistinguishable from HF-2 neurons. $\boldsymbol{A}$, Schematic of the avian song circuit, as in Figure $1 A$. Recording electrodes were chronically implanted into DLM and stimulating electrodes placed in its target, LMAN. $\boldsymbol{B}$, Small electrolytic lesions confirmed the location of the recording electrode in DLM (left) and the stimulating electrode in LMAN (middle). Right, A confocal image of a pallidal axon calyceal terminal (red) and the postsynaptic thalamic neuron (green). C, Antidromic identification and collision testing of LMAN-projecting DLM neurons. $D$, Scatter plot of mean firing rate during singing versus spike duration for all neurons recorded in DLM. Units with spike durations of $<0.06$ ms were identified as pallidal terminals (black circles). Putative DLM units (black circles) and antidromically identified DLM units (green circles) form a distinct cluster. $\boldsymbol{E}-\boldsymbol{K}$, Data are laid out exactly as in Figure $2 E-K$. Compare this pallidal terminal to the HF-2 neuron in Figure 2. Note that power spectrum of the terminals (black) overlaps the HF-2 power spectra $(\boldsymbol{I}, \boldsymbol{J})$, and that the terminals overlap with the HF-2 cluster in peak firing rate and fraction of power below $25 \mathrm{~Hz}(\boldsymbol{K})$.

$\mathrm{dph}$, and one young adult, aged $120 \mathrm{dph}$ ), and, as has been described in anesthetized birds, we encountered two classes of units: DLM neurons and the pallidal axon terminals. These units in DLM were separable by spike duration, mean singing firing rate, and by antidromic activation from LMAN (Fig. 3C,D) (see Materials and Methods).

Pallidal terminals recorded in DLM exhibited singing-related activity that was indistinguishable from HF-2 neurons (Fig. $3 E-H)$. First, terminals did not exhibit bursts (burst rate: $0.02 \pm 0.05 \mathrm{~Hz}$ ), or long pauses (average interval greater than the 99th percentile ISI: $14.6 \pm 5.8 \mathrm{~ms}$ ). Second, terminals exhib-

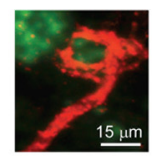

ited increased mean firing rates during singing $(142 \pm 28 \mathrm{~Hz}$ nonsinging vs $290 \pm$ $59 \mathrm{~Hz}$ singing, $p<0.0001$, paired $t$ test $n=37 / 37 ; 11$ birds) (supplemental Fig. 5, available at www.jneurosci.org as supplemental material). As in HF-2 neurons, the firing rate increased gradually before sing$\operatorname{ing}(0.56 \pm 0.22 \mathrm{~s})$, and slowly returned to baseline following singing $(1.64 \pm 1.20 \mathrm{~s})$. During singing, fluctuations in firing rate were extremely rapid, such that the autocorrelation of the instantaneous firing rate during singing was narrow $(5.1 \pm 1.5$ $\mathrm{ms}$ )-slightly longer than the average duration of a single ISI $(3.6 \pm 1.0 \mathrm{~ms})$ (Fig. $3 H$ ).

Notably, in all of these parameters, terminals significantly differed from HF-1 neurons but not from HF-2 neurons ( $p<$ 0.01 vs HF- $1, p>0.1$ vs HF-2 for all unpaired $t$ tests) (Table 1 ). In addition, all pallidal terminals exhibited only weak modulations in the $1-25 \mathrm{~Hz}$ band (Fig. $3 I$ ), and exhibited a distribution of peak firing rates that overlapped entirely with the HF-2 distribution (Fig. $3 K$ ). These data support the hypothesis that HF-2 neurons constitute the thalamus-projecting pallidal neurons in area X. As with HF-1 and HF-2 neurons, the activity of terminals was not different in subsong and plastic song birds (Table 1).

The activity of primate GPe and GPi neurons differs on the same metrics as area X HF-1 and HF-2 neurons

We next wondered whether the features that distinguish thalamus-projecting and putative nonprojecting area $\mathrm{X}$ neurons in the songbird generalize to mammals. To test this hypothesis, we examined the firing patterns of $70 \mathrm{GPe}$ and $59 \mathrm{GPi}$ neurons recorded in the awake behaving primate (see Materials and Methods). We were particularly interested in the possibility that the same analyses that distinguished HF-1 from HF-2 neurons would also distinguish neurons in the GPe from neurons in the GPi.

We examined the IFRs of GPe and GPi neurons and found that, as has been previously described (Elias et al., 2007; Joshua et al., 2009), most GPe neurons exhibited prominent bursts and long pauses (Fig. $4 A, B$ ). In contrast, most GPi neurons discharged continuously (Fig. $4 E, F$ ). As with HF-1 neurons, the large rate modulations of GPe neurons resulted in increased power at low frequencies of their IFR power spectra $(<2 \mathrm{~Hz})$ (Fig. 4I). In fact, GPe neurons were almost completely distinguishable from GPi neurons on the basis of their increased power at low frequencies (Fig. $4 J$, Table 2). We meansubtracted and smoothed the IFRs by low-pass filtering below $2 \mathrm{~Hz}$ (Fig. 4A, B, E, F, blue traces) (see Materials and Methods), and found that GPe neurons were also largely distinguishable 
A

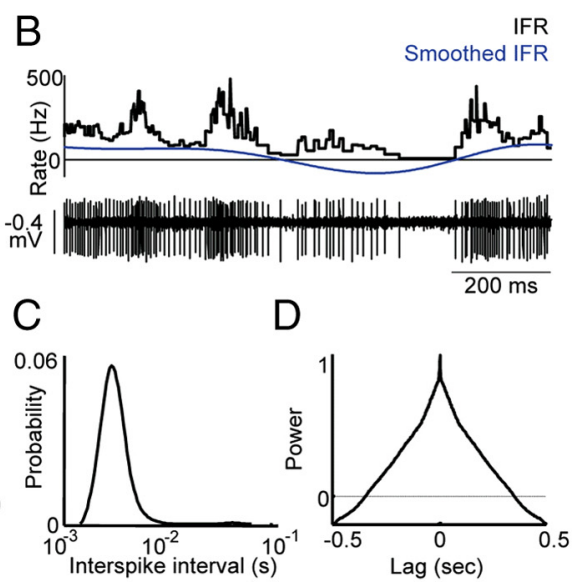

E

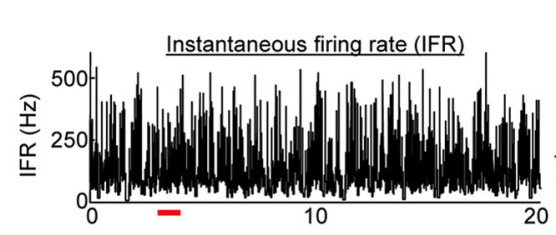

$\mathrm{F}$
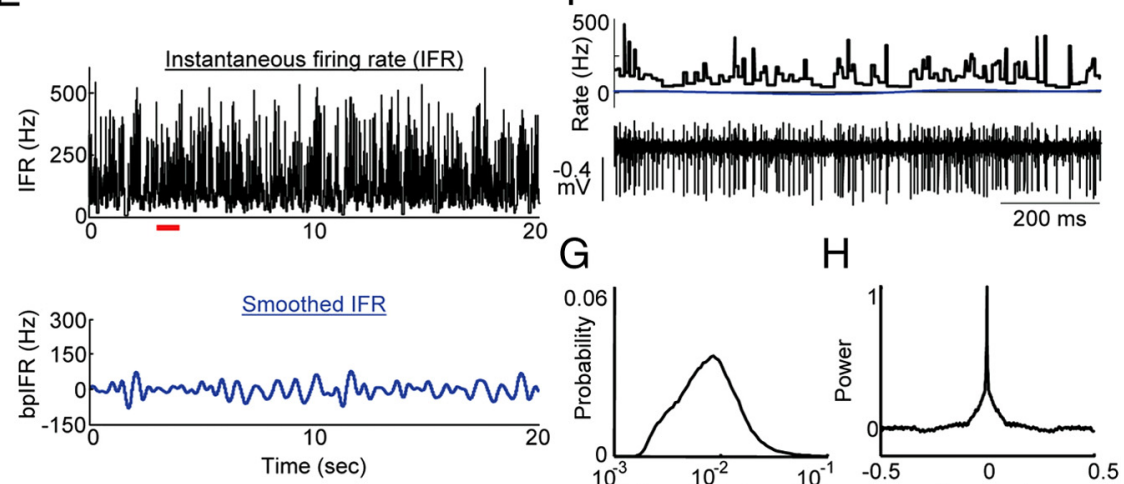

I

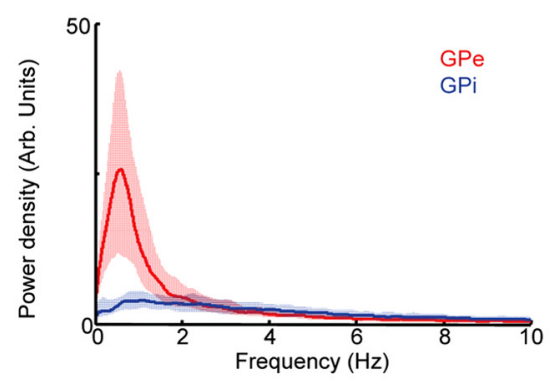

$\mathrm{K}$

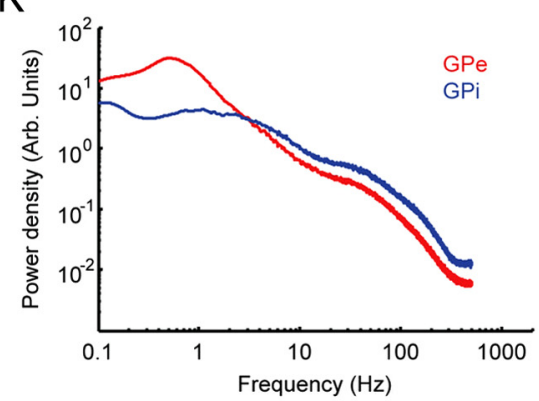

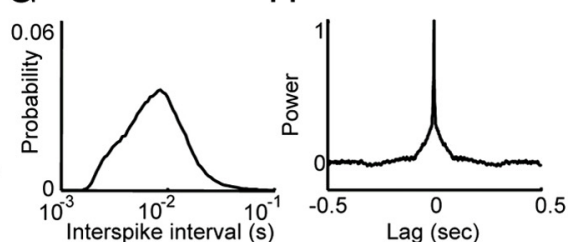

$\mathrm{J}$


$\mathrm{L}$

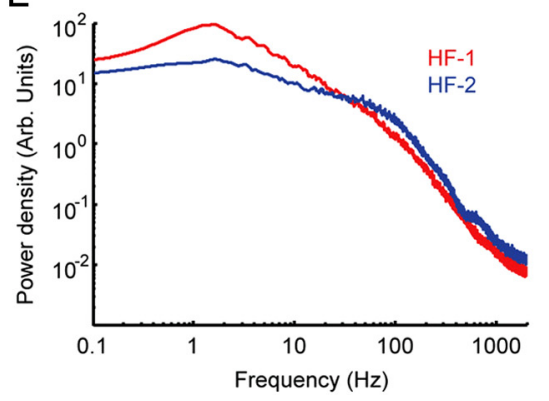

from GPi neurons by their large peaks in the smoothed IFR (Fig. 4J). In fact, with bursts defined as events where the meansubtracted, smoothed IFR exceeds 50 spikes per second (see Materials and Methods), we found that GPe neurons generated bursts significantly more frequently than GPi neurons (GPe: $0.43 \pm$ $0.26 \mathrm{~s}^{-1}$ vs GPi: $0.09 \pm 0.08 \mathrm{~s}^{-1}, p<$ $0.001)$. Thus, an analysis that distinguished HF-1 from HF-2 neurons also distinguished most neurons in the GPe from neurons in the GPi.

Additional well documented differences between GPe and GPi firing patterns were similar to the differences between HF-1 and HF-2 neurons, respectively (DeLong, 1971; Elias et al., 2007). First, GPi neurons exhibited less variability in interspike interval duration than GPe neurons (GPi: C.V. $=0.80 \pm 0.22$; GPe: C.V. $=2.27 \pm 1.00, p<0.001)(\mathrm{Ta}-$ ble 2). Second, firing rate fluctuations in GPi neurons were rapid, such that a given interspike interval was weakly correlated with its neighboring intervals. As a result, the IFR autocorrelation exhibited a significantly narrower peak in GPi neurons than in most GPe neurons (half-width: GPe: $114.4 \pm 90.2 \mathrm{~ms}$, GPi: $9.2 \pm 6.8 \mathrm{~ms}$, $p<0.0001$ unpaired $t$ test) (Fig. $4 D, H$, Table 2). Finally, as has been previously reported, most GPe neurons exhibited more robust pausing in their activity than GPi neurons. For example, intervals greater than 100 ms occurred significantly more frequently in GPe neurons $(0.48 \pm$ $\left.0.30 \mathrm{~s}^{-1}\right)$ than in GPi neurons $(0.17 \pm$ $0.29 \mathrm{~s}^{-1}, p<0.001$, unpaired $t$ test), and pauses, here defined as intervals greater than the 99.9th percentile ISI, were significantly longer in GPe neurons (mean pause duration, GPi: $104.9 \pm 63.4 \mathrm{~ms}$; GPe: $470.7+271.3 \mathrm{~ms}, p<0.001)$ (Table 2).

To further elucidate the relation between firing rate modulations in songbird HF neurons and primate pallidal neurons, we plotted the power spectra of the instantaneous firing rate side by side on log-log plots. Remarkably, the shape of the GPe and GPi power spectra strongly resembled those of HF- 1 and HF-2 neurons. In both

Figure 4. Primate GPe and GPi neurons resemble songbird HF-1 and HF-2 neurons. Data are presented exactly as in Figure 2. $\boldsymbol{A}-\boldsymbol{D}$, Example of a neuron recorded from the GPe of an awake monkey. $\boldsymbol{A}$, The IFR computed from the activity of a single neuron (top), and the same IFR trace smoothed by low-pass filtering below $2 \mathrm{~Hz}$ (bottom). The high mean firing rate was removed (mean-subtracted) using a high-pass filter above $0.1 \mathrm{~Hz}$ (see Materials and Methods). $\boldsymbol{B}$, Expanded view of the IFR (black) and smoothed IFR (blue) from the period indicated by the red bar beneath the IFR trace in $\boldsymbol{A}$. Bottom, The raw voltage data of the isolated neuron. $\boldsymbol{C}, \boldsymbol{D}$, The log of the ISI distributions $(\boldsymbol{C})$ and the lag-autocorrelation function of the IFR $(\boldsymbol{D})$ are plotted for data from the neuron shown in $\boldsymbol{A}$. $\boldsymbol{E}-\boldsymbol{H}$, Data are plotted exactly as in $\boldsymbol{A}-\boldsymbol{D}$, for a neuron recorded from the primate GPi. $\boldsymbol{I}$, The power spectra of the IFRs (median over all cells, shaded areas indicate 25 th to 75 th percentile) for $79 \mathrm{GPe}$ neurons (red) and $50 \mathrm{GPi}$ neurons (blue). Note that the IFRs of GPe neurons exhibit more power at low frequencies $(<2 \mathrm{~Hz}) . J$, For each GPe neuron and GPi neuron recorded, the percentage of the IFR power spectrum at low frequencies $(<2 \mathrm{~Hz})$ is plotted against the peak (99th percentile) of the

$\leftarrow$

mean-subtracted, smoothed IFR. At bottom, the histogram of peak values of the smoothed IFR and, at left, the histogram of fraction of IFR power below $2 \mathrm{~Hz}$. Note that these metrics distinguish GPi neurons from most GPe neurons. $\boldsymbol{K}, \boldsymbol{L}$, Side-byside comparison of the complete IFR power spectra, plotted on $\log -\log$ axes, of all GPe and GPi neurons ( $\boldsymbol{K}$ ) and all HF-1 and HF-2 neurons from the songbird $(L)$. Data show the average spectrum over all neurons within each group. 
Table 2. Spiking statistics of primate GPe and GPi neurons

\begin{tabular}{|c|c|c|c|c|c|c|c|}
\hline & Rate (Hz) & C.V.ISI & $\begin{array}{l}\text { 99th percentile } \\
\text { ISI (ms) }\end{array}$ & $\begin{array}{l}\text { 99.9th percentile } \\
\text { ISI (ms) }\end{array}$ & $\begin{array}{l}\text { IFR autocorrelation } \\
\text { half-width (ms) }\end{array}$ & $\begin{array}{l}\% \text { of IFR spectrum in } \\
\text { the } 0.1-4 \mathrm{~Hz} \text { band }\end{array}$ & $\begin{array}{l}\% \text { of IFR spectrum in } \\
\text { the } 1-25 \mathrm{~Hz} \text { band }\end{array}$ \\
\hline $\mathrm{GPe}(n=70)$ & $84.0 \pm 23.4$ & $2.27 \pm 1.00$ & $39.6 \pm 25.1$ & $470.7 \pm 271.3$ & $114.4 \pm 90.2$ & $3.9 \pm 1.2$ & $8.8 \pm 1.6$ \\
\hline $\mathrm{GPi}(n=59)$ & $88.7 \pm 23.6$ & $0.80 \pm 0.22$ & $11.9 \pm 5.2$ & $104.9 \pm 63.4$ & $9.2 \pm 6.8$ & $1.5 \pm 0.4$ & $6.2 \pm 1.3$ \\
\hline Interclass comparisons & n.s. & $\mathrm{GPe}>\mathrm{GPi}^{*}$ & n.s. & $\mathrm{GPe}>\mathrm{GPi}^{*}$ & $\mathrm{GPe}>\mathrm{GPi}^{*}$ & $\mathrm{GPe}>\mathrm{GPi}^{*}$ & $\mathrm{GPe}>\mathrm{GPi}^{*}$ \\
\hline
\end{tabular}

Interclass comparisons indicate significant differences between cell classes ( $p<0.01)$. Asterisks indicate the metrics that distinguished, as a group, GPe from GPi neurons as well as HF-1 from HF- 2 neurons and terminals.

the primate and the songbird, HF-1 neurons and GPe neurons exhibited increased fluctuations in firing rate at low modulation frequencies compared to their HF-2 and GPi counterparts. Furthermore, in both songbird and primate, the firing rate modulations of HF-1 and GPe neurons fell off rapidly at higher frequencies such that HF-2 and GPi neurons exhibited more modulation at frequencies above some cross-over point. This cross-over happened at $\sim 2.5 \mathrm{~Hz}$ in primates, and at $\sim 25 \mathrm{~Hz}$ in the zebra finch (Fig. $4 K, L$ ).

Thus, while primate pallidal neurons exhibited slower dynamics than area X HF neurons, including lower firing rates, longer pauses, and more power distributed at lower frequencies in their IFR spectra (Tables 1, 2, Figs. 3I, 4I), the differences between GPe and GPi neurons paralleled the differences between HF-1 and HF-2 neurons in all of the features examined (Tables 1, 2 ). These data raise the possibility that low-frequency modulations, including bursts and long pauses, are general features of pallidal neurons that primarily do not project to the thalamus, while continuous spiking without long bursts and long pauses is a general feature of thalamus-projecting pallidal neurons.

\section{Correlations of firing patterns with syllable temporal structure}

Several additional features of pallidal firing patterns in primates were also observed in HF neurons recorded in area X. First, pallidal neurons exhibit firing rate modulations that are time locked to behavioral tasks (DeLong, 1971; Arkadir et al., 2004; Turner and Anderson, 2005; Joshua et al., 2009). To determine whether HF neurons also exhibited "task-related" correlations, we examined spike rasters aligned to song. Song-aligned rate histograms revealed that both rate increases (peaks) and rate decreases (minima) could be significantly correlated with song temporal structure (Fig. 5A-F) (see Materials and Methods for assessment of statistical significance). In fact, correlations to song timing were pervasive: almost every neuron exhibited one or more significant rate peaks (or minima) during every syllable in the bird's repertoire $(n=66 / 67$ syllables in HF-1, 44/45 syllables in HF-2, 81/82 syllables in terminals).

Second, it has been noted that individual GPe neurons exhibit a broad range of firing rates during a motor task, and that the depth of these rate modulations is larger in GPe than in GPi neurons (Turner and Anderson, 1997; Joshua et al., 2009). From the rate histograms, we computed the modulation depths for each neuron during each syllable, defined as the peak-tominimum difference in the rate modulations relative to the mean rate during that syllable (see Materials and Methods). We found that modulation depths in HF-1 neurons were, on average, significantly larger than HF-2 neurons and terminals (Fig. 5G) (HF-1: 0.34 ..17, HF-2: $0.12 \pm 0.05$ and terminals: $0.14 \pm .07, p<0.01$ HF-1 vs HF-2 and terminals, $p>0.05$ HF-2 vs terminals).

Third, both significant increases and decreases in the firing rates of primate pallidal neurons can be distributed throughout multiple stages of a sequential motor task (Arkadir et al., 2004). We found for HF-1, HF-2, and terminals that both statistically significant firing rate peaks in the average rate histograms and significant firing rate minima were distributed throughout the song and were not clustered around syllable onsets or offsets (Fig. $5 H, I$; supplemental Fig. 6, available at www.jneurosci.org as supplemental material) (see Materials and Methods).

Finally, many primate pallidal neurons can exhibit trial-totrial variability in the timing of their activity during motor tasks (Turner and Anderson, 2005). Similarly, the firing rate modulations of HF neurons exhibited trial-to-trial variability across syllable renditions. In fact, CCs computed between spike trains on a trial (syllables)-by-trial basis were significant but low (HF-1: $0.047 \pm 0.045$; HF-2: $0.034 \pm 0.027$; terminals: $0.050 \pm 0.45$, see Materials and Methods). Next, because HF-1 neurons exhibited two distinct modes of firing - bursts and pauses-we wondered whether there was any difference in the relative temporal precision of burst and pause events. We computed cross-correlation coefficients between burst events, and, for comparison, between pause events, in all pairs of trials, and found that both bursts and pauses could be significantly correlated to song timing (burst CC: $0.046 \pm 0.063$, significant in 37/65 syllables; pause CC: $0.12 \pm$ 0.12 , significant in 33/50 syllables, see Materials and Methods). However, the timing of pauses was on average more reliable over repeated syllable renditions than the timing of bursts (supplemental Fig. 7, available at www.jneurosci.org as supplemental material) $(p<0.001$, paired test $)$.

\section{Spike correlations between simultaneously recorded pairs of area $X$ output neurons}

HF-2 neurons and terminals discharged at high rates with a relatively narrow distribution of interspike intervals, especially during nonsinging periods (Fig. 2). In mammals it has been shown that GPi neurons, which exhibit similar firing patterns, are not synchronized with one another, exhibiting flat spike train crosscorrelations between simultaneously recorded neurons (Nini et al., 1995). Although it was difficult to record more than one neuron at the same time, our dataset included four simultaneously recorded pairs of area $\mathrm{X}$ output neurons (supplemental Fig. $8 A$, available at www.jneurosci.org as supplemental material) ( $n=2$ pairs of terminals and $n=2$ pairs of HF-2 neurons). We calculated spike train cross-correlograms (bin size $2 \mathrm{~ms}$ ) and found that they did not exhibit strongly correlated spike times, as evidenced by flat cross-correlograms without significant peaks or minima (supplemental Fig. $8 B, C$, available at www.jneurosci.org as supplemental material) (see Materials and Methods). Thus, similar to what has been observed in the primate GPi (Nini et al., 1995), in this limited dataset area X output neurons were not synchronized. Of course, the absence of a significant peak in these four pairs does not rule out the possibility that some area $\mathrm{X}$ neurons exhibit synchronous firing.

\section{Discussion}

The existence of direct and indirect striatopallidal pathways is a highly conserved feature of basal ganglia circuitry (Reiner, 2009). It has long been known that in awake mammals, most output 
pallidal neurons that project to the thalamus (GPi) exhibit firing patterns that are distinguishable from pallidal neurons that project primarily within the BG circuit (GPe) (DeLong, 1971). To determine whether these differences represent a recent adaptation specific to mammals or whether they are essential features of BG function that predate the division of mammals, reptiles, and birds into distinct classes, we recorded from putatively homologous pallidal cell classes in the songbird area $\mathrm{X}$, and compared their firing patterns to neurons recorded in the primate globus pallidus.

\section{$\mathrm{HF}$ units in area $\mathrm{X}$ are putative pallidal neurons}

In mammals and in songbird area $\mathrm{X}$, the hallmark feature distinguishing pallidal from striatal cell types is their high spontaneous firing rate $(>60 \mathrm{~Hz}$ ) (DeLong, 1972; Farries and Perkel, 2002; Leblois et al., 2009). In the present study, we suggest this distinction also applies to neurons in area $\mathrm{X}$ of awake birds (Fig. 1), and hypothesize that the high-firing-rate units correspond to pallidal neurons, while the low-firing units are striatal. This hypothesis was strengthened by the finding that all pallidal terminals recorded in DLM $(n=$ 37/37) exhibited spontaneous firing rates entirely within the distribution of $\mathrm{HF}$ neurons in area X (Table 1). All terminals gradually increased their rates before singing, fired continuously at high rates during singing without prominent bursts or long pauses, and gradually returned to baseline (Fig. 3). Because HF-2 neurons behaved identically to terminals (Figs. 2, 3; supplemental Figs. 2, 3, available at www.jneurosci.org as supplemental material), we propose that HF-2 neurons, and those recorded in area $\mathrm{X}$ of a previous study (Hessler and Doupe, 1999), constitute DLM-projecting neurons in area X. Pallidal terminals recorded in DLM never exhibited the singing-related burst and pausing behavior observed in HF-1 neurons, suggesting that HF-1 neurons constitute the pallidal neurons in area $\mathrm{X}$ that do not project to DLM. Future studies with intracellular labeling of HF neurons in singing birds could confirm this hypothesis.

\section{Comparison of HF neurons in area $\mathrm{X}$} with primate pallidal neurons

In mammals, the pallidal neurons that project to the ventral thalamus and those that do not are anatomically segregated (the GPi and GPe of primates). Although both pallidal cell types exhibit high spontaneous firing rates, several differences in their firing patterns have been noted: most GPe neurons tend to exhibit

C
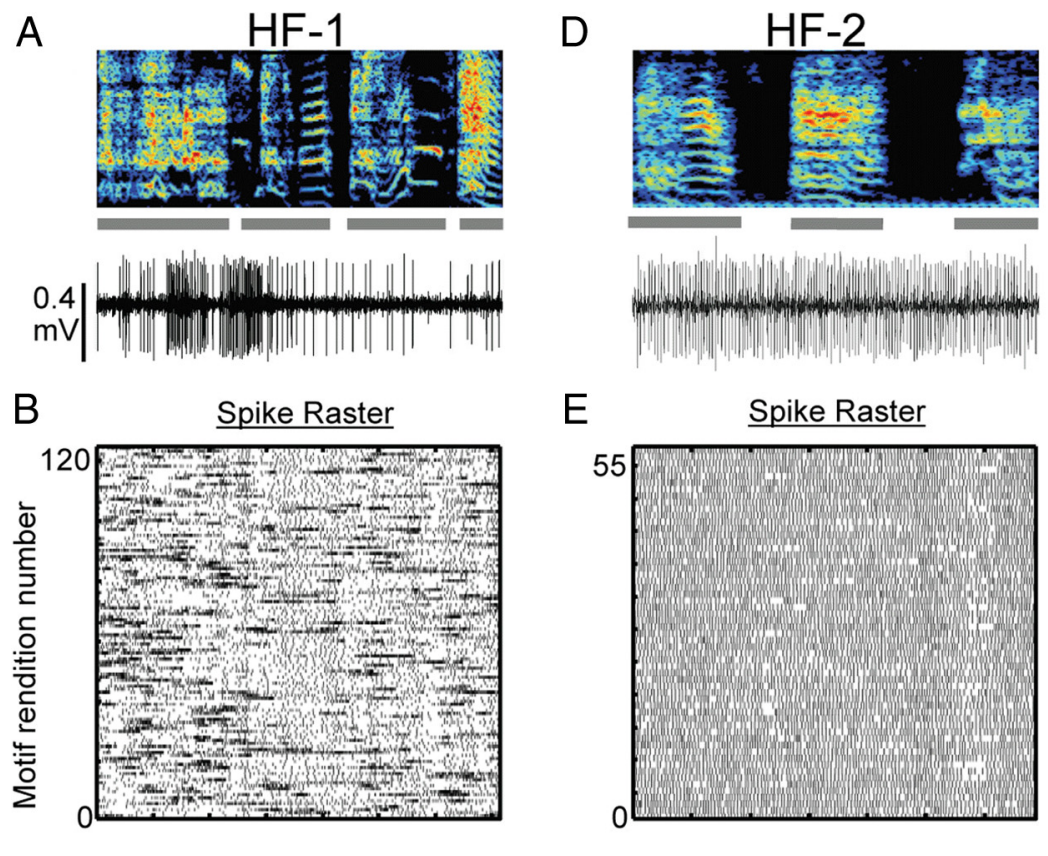

E
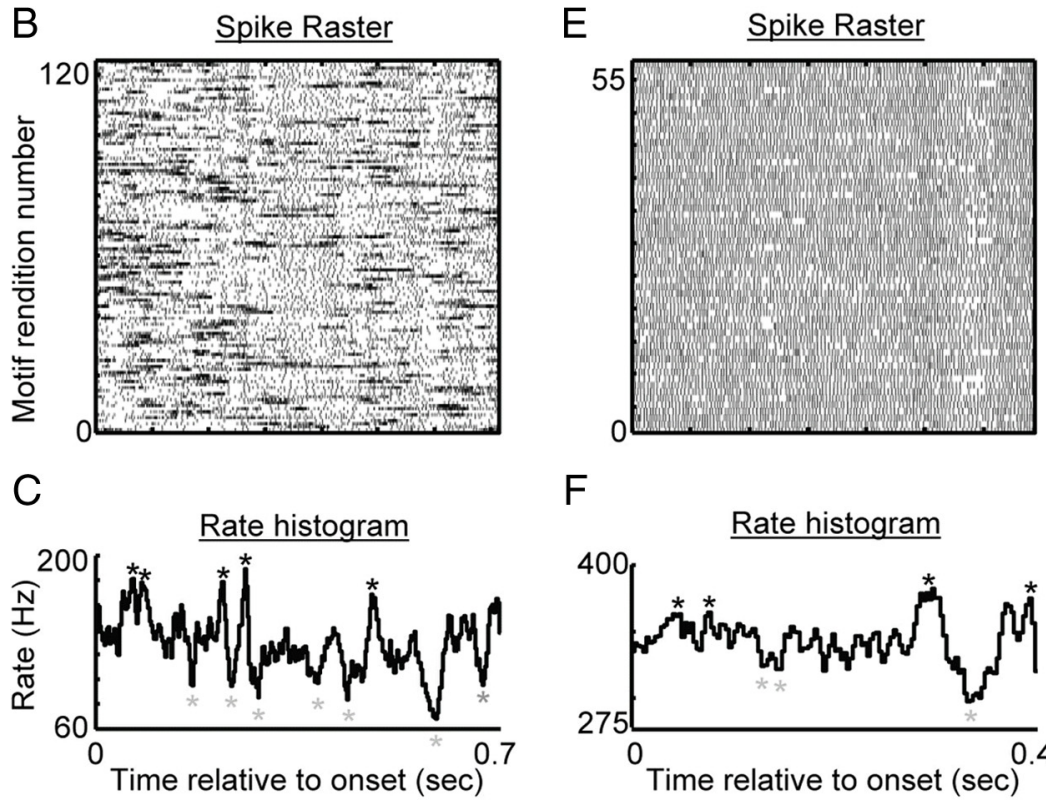

F
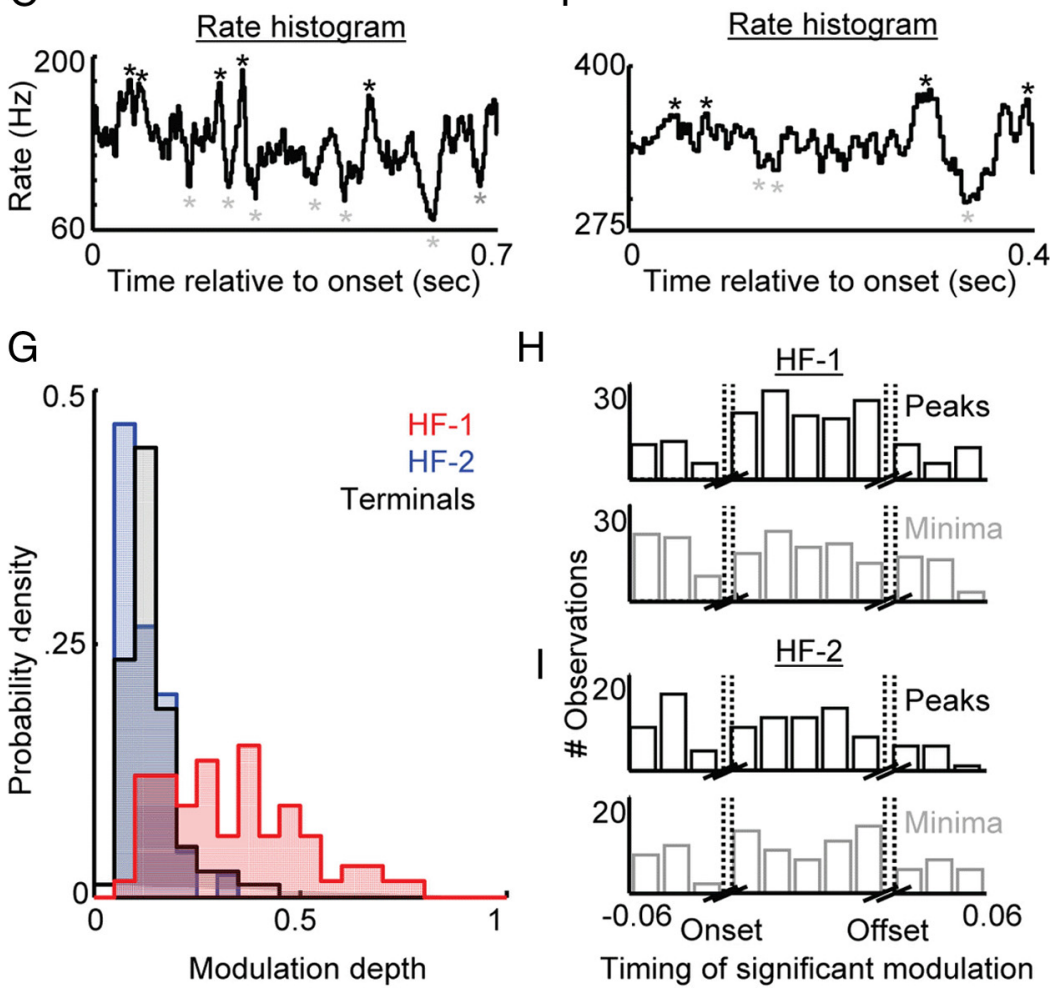

$\mathrm{H}$
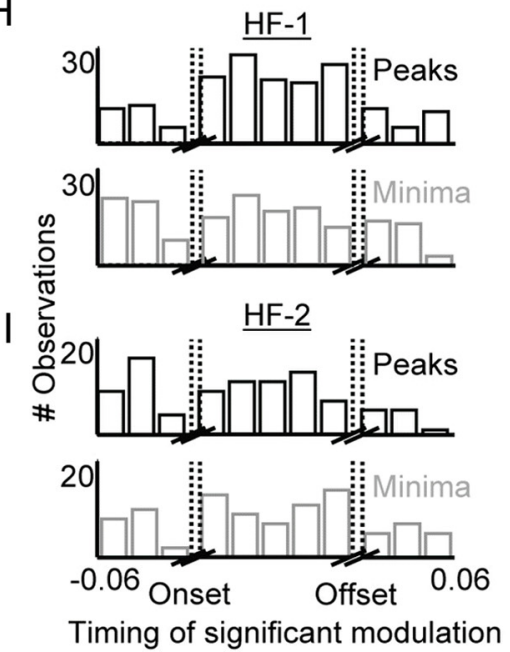

Figure 5. The activity of HF neurons in area X is modulated by syllable timing. $A$, Voltage waveform of an HF-1 neuron is plotted beneath the time-aligned song spectrogram. $\boldsymbol{B}$, Raster plot of spiking activity during 122 consecutive motif renditions. $\boldsymbol{C}$, Rate histogram compiled from the raster plot. Asterisks indicate significant rate peaks (black) and minima (gray) in firing rate. Time axis in $\boldsymbol{C}$ also applies to $\boldsymbol{A}$ and $\boldsymbol{B}$. D-F, Data plotted as in $\boldsymbol{A}-\boldsymbol{C}$ for an HF-2 neuron. $\boldsymbol{G}$, Histogram of the modulation depths observed during all syllables for all HF-1 neurons (red), HF-2 neurons (blue), and pallidal terminals (black). $\boldsymbol{H}, \boldsymbol{I}$, Histograms showing the incidence of significant peaks (top, black) and minima (bottom, gray) in HF-1 neurons $(\boldsymbol{H})$ and HF-2 neurons ( $\boldsymbol{I}$ ). Note that significant modulations occur throughout the syllables. longer interspike intervals (pauses) and slower modulations in their firing rates (DeLong, 1971). In amphibians, reptiles, and birds, the two pallidal cell types are intermingled, but it has remained unknown whether this intermingling is associated with an absence of distinct firing patterns (Reiner, 2009). Do the dis- 
tinct firing patterns of GPe- and GPi-like pallidal neurons arise during evolution in tandem with their anatomical segregation? To our knowledge, this is the first study to address this question, and we suggest that despite their colocalization in area $\mathrm{X}$, thalamus-projecting (HF-2 and terminals) and putative nonprojecting (HF-1) pallidal neurons exhibit distinct firing patterns during behavior.

Intriguingly, the features that distinguished HF-1 from HF-2 and terminal firing patterns also distinguished most neurons in the GPe from those in the GPi (Fig. 4). Like HF-2 neurons and terminals, GPi neurons typically discharged continuously without long intervals of silence and showed considerable irregularity with frequent brief fluctuations in rate (Fig. 4). In contrast, HF-1 neurons and most GPe neurons exhibited slower modulations in firing rate, including long pauses and bursts. As a result, several measures of neural activity-pause duration, burst rate, percentage of the IFR spectra in a low-frequency band, and half-width of IFR autocorrelation-were larger in the GPe than in GPi, and also larger in HF-1 neurons than in HF-2 neurons and terminals (Tables 1, 2).

Despite these similarities, there were also substantial differences between the firing patterns of HF neurons in area $\mathrm{X}$ and primate pallidal neurons we recorded. Primate pallidal neurons exhibited significantly slower firing rate modulations than HF neurons. First, the firing rates of HF neurons (higher than $\sim 150$ $\mathrm{Hz}$ during singing) were higher than what was observed in the primate pallidus $(\sim 80 \mathrm{~Hz})$ (Tables 1,2$)$. Second, pauses in GPe neurons $(\sim 470 \mathrm{~ms})$ were significantly longer than in HF-1 neurons $(\sim 50 \mathrm{~ms})$. Third, although GPe neurons frequently exhibited bursts in between long pauses, the large, singing-related burst events generated by HF-1 neurons were not typical in GPe neurons. Indeed, traditionally pauses, but not bursts, have been used as a feature to differentiate GPe from GPi neurons. In principle, many of these differences could be attributable to the higher temperature of songbirds, or to the fact that the primate pallidal neurons have not been examined in a behavioral task that requires the fast motor timescales of singing. However, they may also signify interspecies differences in pallidal signaling.

In addition, although the basic wiring diagram of the anterior forebrain pathway (AFP) is similar to mammalian BG-thalamocortical loops (Fig. 1), a major difference is that the mammalian indirect pathway includes a relay through the subthalamic nucleus (STN), a glutamatergic structure functionally interposed between the external and internal pallidal segments. While birds do have an STN (Jiao et al., 2000), it does not appear to be interconnected with area $\mathrm{X}$, suggesting that either the STN is not required to generate distinct firing patterns in thalamusprojecting and nonprojecting pallidal cell types (Nambu et al., 2000), or the functionality of the STN is performed by circuit elements within area $\mathrm{X}$, such as direct excitatory projections to pallidal neurons from HVC and LMAN (Farries et al., 2005).

\section{Relationship of neural activity to singing behavior}

Several features of the correlations observed in HF neurons in this study are similar to what has been observed in mammalian pallidal neurons in other studies. First, primate pallidal neurons can encode the timing of task events with either rate increases or decreases, and at multiple times within a task (Arkadir et al., 2004). Similarly, significant rate modulations occurred in HF neurons throughout the song (Fig. 5). Second, mammalian pallidal neurons can exhibit significant trial-to-trial variability in their spike timing (Turner and Anderson, 1997), although the function of this variability remains unclear. HF neurons were also variable in their spiking, exhibiting correlation coefficients similar to what has been observed in LMAN, a frontal cortical homolog that forms the output of the AFP. LMAN drives vocal variability (Kao et al., 2005; Olveczky et al., 2005) and also generates an error-related signal that biases vocal output to improve performance (Andalman and Fee, 2009). The correlations to song timing observed in this study (Fig. 5) may be a neural fingerprint underlying this premotor bias.

\section{Summary}

We have recorded pallidal axon terminals in the thalamic nucleus DLM, as well as high-firing-rate neurons in the striatopallidal nucleus area $\mathrm{X}$, and report two HF cell classes. HF-1 neurons exhibit singing-related bursts and large modulations in firing rate, while HF-2 neurons did not burst and exhibited continuous discharge. All pallidal terminals recorded in DLM produced firing patterns indistinguishable from HF-2 neurons (Figs. 2, 3, Table 1). This led us to hypothesize that HF-1 neurons, putative nonprojecting pallidal neurons, were functionally homologous to GPe neurons, while HF-2 neurons and terminals were functionally homologous to thalamus-projecting neurons in the GPi. To directly test this hypothesis, we analyzed data recorded in awake behaving primates, and found that most GPe neurons differed from GPi neurons in the same measures used to separate HF-1 from HF-2 neurons (Fig. 4). Our data suggest that the conserved structural organization of two pallidal pathways in the basal ganglia of mammals and non-mammals extends to the types of functional signals they generate.

\section{References}

Andalman AS, Fee MS (2009) A basal ganglia-forebrain circuit in the songbird biases motor output to avoid vocal errors. Proc Natl Acad Sci U S A 106:12518-12523.

Arkadir D, Morris G, Vaadia E, Bergman H (2004) Independent coding of movement direction and reward prediction by single pallidal neurons. J Neurosci 24:10047-10056.

Bar-Gad I, Heimer G, Ritov Y, Bergman H (2003) Functional correlations between neighboring neurons in the primate globus pallidus are weak or nonexistent. J Neurosci 23:4012-4016.

DeLong MR (1971) Activity of pallidal neurons during movement. J Neurophysiol 34:414-427.

DeLong MR (1972) Activity of basal ganglia neurons during movement. Brain Res 40:127-135.

Doupe AJ, Perkel DJ, Reiner A, Stern EA (2005) Birdbrains could teach basal ganglia research a new song. Trends Neurosci 28:353-363.

Elias S, Joshua M, Goldberg JA, Heimer G, Arkadir D, Morris G, Bergman H (2007) Statistical properties of pauses of the high-frequency discharge neurons in the external segment of the globus pallidus. J Neurosci 27:2525-2538.

Farries MA, Perkel DJ (2002) A telencephalic nucleus essential for song learning contains neurons with physiological characteristics of both striatum and globus pallidus. J Neurosci 22:3776-3787.

Farries MA, Ding L, Perkel DJ (2005) Evidence for "direct" and "indirect" pathways through the song system basal ganglia. J Comp Neurol 484:93-104.

Fee MS, Leonardo A (2001) Miniature motorized microdrive and commutator system for chronic neural recording in small animals. J Neurosci Methods 112:83-94.

Fee MS, Mitra PP, Kleinfeld D (1997) Central versus peripheral determinants of patterned spike activity in rat vibrissa cortex during whisking. J Neurophysiol 78:1144-1149.

Goldberg JH, Fee MS (2010) Singing-related neural activity distinguishes four classes of putative striatal neurons in the songbird basal ganglia. J Neurophysiol 103:2002-2014.

Hahnloser RH, Kozhevnikov AA, Fee MS (2002) An ultra-sparse code underlies the generation of neural sequences in a songbird. Nature 419:65-70.

Hessler NA, Doupe AJ (1999) Singing-related neural activity in a dorsal 
forebrain-basal ganglia circuit of adult zebra finches. J Neurosci 19:10461-10481.

Jiao Y, Medina L, Veenman CL, Toledo C, Puelles L, Reiner A (2000) Identification of the anterior nucleus of the ansa lenticularis in birds as the homolog of the mammalian subthalamic nucleus. J Neurosci 20:6998-7010.

Johnstone S, Rolls ET (1990) Delay, discriminatory, and modality specific neurons in striatum and pallidum during short-term memory tasks. Brain Res 522:147-151.

Joshua M, Adler A, Rosin B, Vaadia E, Bergman H (2009) Encoding of probabilistic rewarding and aversive events by pallidal and nigral neurons. J Neurophysiol 101:758-772.

Kao MH, Doupe AJ, Brainard MS (2005) Contributions of an avian basal ganglia-forebrain circuit to real-time modulation of song. Nature 433:638-643

Kao MH, Wright BD, Doupe AJ (2008) Neurons in a forebrain nucleus required for vocal plasticity rapidly switch between precise firing and variable bursting depending on social context. J Neurosci 28: 13232-13247.

Kojima S, Doupe AJ (2009) Activity propagation in an avian basal gangliathalamocortical circuit essential for vocal learning. J Neurosci 29:4782-4793.

Leblois A, Bodor AL, Person AL, Perkel DJ (2009) Millisecond timescale disinhibition mediates fast information transmission through an avian basal ganglia loop. J Neurosci 29:15420-15433.
Nambu A, Tokuno H, Hamada I, Kita H, Imanishi M, Akazawa T, Ikeuchi Y, Hasegawa N (2000) Excitatory cortical inputs to pallidal neurons via the subthalamic nucleus in the monkey. J Neurophysiol 84:289-300.

Nini A, Feingold A, Slovin H, Bergman H (1995) Neurons in the globus pallidus do not show correlated activity in the normal monkey, but phase-locked oscillations appear in the MPTP model of parkinsonism. J Neurophysiol 74:1800-1805.

Olveczky BP, Andalman AS, Fee MS (2005) Vocal experimentation in the juvenile songbird requires a basal ganglia circuit. PLoS Biol 3:e153.

Person AL, Perkel DJ (2007) Pallidal neuron activity increases during sensory relay through thalamus in a songbird circuit essential for learning. J Neurosci 27:8687-8698.

Reiner A (2009) You cannot have a vertebrate brain without a basal ganglia. In: The basal ganglia IX, pp 3-24. New York: Springer.

Smeets WJ, Marín O, González A (2000) Evolution of the basal ganglia: new perspectives through a comparative approach. J Anat 196:501-517.

Turner RS, Anderson ME (1997) Pallidal discharge related to the kinematics of reaching movements in two dimensions. J Neurophysiol 77:10511074.

Turner RS, Anderson ME (2005) Context-dependent modulation of movement-related discharge in the primate globus pallidus. J Neurosci 25:2965-2976. 